\title{
Scale-bridging phase-field simulations of microstructure responses on nucleation in metals and colloids
}

\author{
Marco Berghoffa and Britta Nestler \\ Institute of Applied Materials, Karlsruhe Institute of Technology
}

\begin{abstract}
In the present studies we investigate the connection between atomistic simulation methods, i. e. molecular dynamics (MD) and phasefield crystal (PFC), to the mesoscopic phase-field methods(PFM). While the first describes the evolution of a system on the basis of motion equations of particles the second uses a Cahn-Hilliard type equation to described an atomic density field and the third grounds on the evolution of continuous local order parameter field. The first aim is to point out the ability of the mesoscopic phase-field method to make predictions of growth velocity at the nanoscopic length scale. Therefore the isothermal growth of a spherical crystalline cluster embedded in a melt is considered. We also show simulation techniques that enable to computationally bridge from the atomistic up to the mesoscopic scale. We use a PFM to simulate symmetric thermal dendrites started at an early stage of solidification related to nucleation. These techniques allow to simulate three dimensional dendrites from the state of nuclei $(\approx 50 \AA)$ converted from MD up to a size of some $\mu \mathrm{m}$ where ternary side-arms start to grow.
\end{abstract}

\section{Introduction}

Computer-aided scientific investigations of materials are carried out on the atomic and mesoscopic scale, to capture the physical processes acting on both scales [1] and to track the transfer of properties across the scales. An understanding of the effects on each scale allows to transfer information, physical properties across different, well-established methods. Bragard et al. for instance, use parameters obtained from molecular dynamics (MD) simulations, to compute nickel dendrites on a micrometer length scale with the phase-field method (PFM). Provatas et al. [2] use a phase-field crystal (PFC) model on the atomistic length scale as an alternative concept, compared to $\mathrm{MD}$, in order to provide input data for PFM simulations [3, 4], whereby PFC can be regarded as the time-related averaging of the particle position in MD [5].

On the atomistic scale, MD is a widely used computational method to describe the atom positions and ordering mechanisms, however, it has high computational effort, such that only small simulations are practicable [6-10]. In recent years, PFC type approaches are developed and contribute to the variety of atomistic material modelling

\footnotetext{
a e-mail: marco.berghoff@kit.edu
} 
methods [11-16]. In contrast to MD, they build on a minimization of atomic energy density functionals and promise to represent the dynamics in a temporal range some orders of magnitude larger than classical MD. PFM usually acts on the mesoscopic scale of several micrometers and is applied to compute microstructure evolution such as dendrites, eutectic or peritectic patterns, (e.g. [17-21]) at which an atomic resolution is no longer possible. Over almost two decades, various model derivatives are formulated so as to study solidification in pure and multi-component systems, e.g. [22-26], microstructure evolution in solid phase systems [27, 28], and general motion of grain boundaries, e.g. [29].

In the next section, we introduce the PFM. In section 3, we describe the conversion from the atom positions and their local order parameter in MD to an interpolated continuous field in PFM, as well as from MD through PFC to PFM. In section 4, we subsequently show an application for pure metals, where pure nickel MD data [30] are mapped into the continuous PFM format. To illustrate the path, we set a nucleus of $50 \AA$ diameter in an undercooled melt. On the atomic scale, we compare the growth of nickel nucleus predicted by both methods: MD and PFC. With the PFM, the calculation of the nucleus is continued, until it results in a dendrite with ternary side arms. In section 5 , we return to the atomic scale and present the PFC model, which is also used for the calculation of colloids. In section 6 , we conclude by carving out the results.

\section{Phase-Field Model}

The growth process in the PFM is modelled by the variables of the inner energy $e$, and two order parameters, $\phi_{s}$ (solid) and $\phi_{\ell}$ (liquid), called phase-fields. In a two phase system, the phase-field variables fulfill the constraint $\phi_{s}+\phi_{\ell}=1$, so that a single phase-field variable $\phi=\phi_{s}$ is sufficient to describe the evolution of the phase boundaries in the system. The variable $\phi(\mathbf{x}, t)$ denotes the local fraction of the considered phase.

The phase-field model is based on an entropy density functional, to ensure consistency with classical, irreversible thermodynamics

$$
\mathcal{S}(e, \phi)=\int_{\Omega} s(e, \phi)-\left(\epsilon a(\nabla \phi)+\frac{1}{\epsilon} w(\phi)\right) \mathrm{d} x .
$$

The bulk entropy density $s$ depends on the internal energy density $e$ and the phasefield variable $\phi$. The functions $a(\nabla \phi)$ and $w(\phi)$ reflect the thermodynamics of the interfaces, and $\epsilon$ is a small length scale parameter related to the thickness of the diffuse interface.

Taking the functional derivatives of Eq. (1) results to the dynamic equations for the non-conserved phase-field variable $\phi$ and the conservative energy

$$
\begin{aligned}
\tau \epsilon \frac{\partial \phi}{\partial t} & =\frac{\delta \mathcal{S}}{\delta \phi}, \\
\frac{\partial e}{\partial t} & =-\nabla \cdot L_{00}(T, \phi) \nabla \frac{\delta \mathcal{S}}{\delta e},
\end{aligned}
$$

where $\tau$ is a kinetic mobility. In case of temperature dependent and anisotropic kinetics, $\tau$ depends on the temperature and the orientation of the phase boundary, i.e. $\tau=\tau(T, \nabla \phi)$. The mobility coefficient $L_{00}(T, \phi)$ is related to the thermal conductivity $K(\phi)$. It is simplified to the expression $L_{00}=K T^{2}$ for constant $K(\phi)=K$. 
The evolution of the phase-field variable is described by

$$
\tau \epsilon \frac{\partial \phi}{\partial t}=\epsilon \nabla \cdot \partial_{\nabla \phi} a(\nabla \phi)-\frac{1}{\epsilon} \partial_{\phi} w(\phi)-\frac{\partial_{\phi} f(T, \phi)}{T},
$$

where $\partial_{\nabla \phi}$ and $\partial_{\phi}$ denote the partial derivatives with respect to $\nabla \phi$ and $\phi$. The bulk free energy density reads

$$
f(T, \phi)=L^{s}(T) \frac{T-T_{m}}{T_{m}} h\left(\phi_{s}\right)+\sum_{\alpha \in\{s, \ell\}}\left(\int_{T_{m}}^{T} c_{v}^{\alpha}(\tilde{T}) \mathrm{d} \tilde{T}-T \int_{T_{m}}^{T} c_{v}^{\alpha}(\tilde{T}) \frac{\mathrm{d} \tilde{T}}{\tilde{T}}\right) h\left(\phi_{\alpha}\right),
$$

where $h(\phi)$ is a monotonic function on $[0,1]$ with $h(0)=0, h(1)=1$ and $h^{\prime}(0)=$ $h^{\prime}(1)=0 . L^{s}(T)$ is the latent heat and $c_{v}^{\alpha}(T)$ is the volumetric heat capacity for solid $(\alpha=s)$ and liquid $(\alpha=\ell)$, which both depend on the temperature $T . T_{m}$ is the melting temperature.

We further define $w(\phi)=\frac{16}{\pi^{2}} \gamma_{s \ell} \phi(1-\phi)$ for $\phi \in[0,1]$ and elsewhere $w(\phi)=\infty$ as an obstacle type potential and $a(\nabla \phi)=\gamma_{s \ell} \gamma(\hat{n})^{2}|\nabla \phi|^{2}$ as the gradient energy density with the anisotropic interface free energy $\gamma$ from MD. We used the notation $\hat{n}=\frac{\nabla \phi}{|\nabla \phi|}$.

The kinetic coefficient $\tau$ depends on the temperature by

$$
\tau(T, \nabla \phi)=\frac{L^{\alpha}(T)}{T T_{m} k(\hat{n})},
$$

with an anisotropic kinetic parameter $k$ provided from the MD model EAM F85.

The evolution of the temperature can be derived from the thermodynamic relation $e=f+T S$ and the entropy $S=-\frac{\partial f}{\partial T}$ by

$$
e(T, \phi)=f(T, \phi)-T \frac{\partial f(T, \phi)}{\partial T}
$$

with $T(\mathbf{x}, t)$ and $\phi(\mathbf{x}, t)$, which are both functions in space and time.

Subsequently, the time derivative of $e$ follows as

$$
\begin{aligned}
\frac{\partial e}{\partial t} & =\frac{\partial f}{\partial T} \frac{\partial T}{\partial t}+\frac{\partial f}{\partial \phi} \frac{\partial \phi}{\partial t}-\frac{\partial T}{\partial t} \frac{\partial f}{\partial T}-T\left(\frac{\partial^{2} f}{\partial T^{2}} \frac{\partial T}{\partial t}+\frac{\partial^{2} f}{\partial T \partial \phi} \frac{\partial \phi}{\partial t}\right) \\
& =\left(-T \frac{\partial^{2} f}{\partial T^{2}}\right) \frac{\partial T}{\partial t}+\left(\frac{\partial f}{\partial \phi}-T \frac{\partial^{2} f}{\partial T \partial \phi}\right) \frac{\partial \phi}{\partial t} .
\end{aligned}
$$

With $\frac{\delta S}{\delta e}=\frac{1}{T}$ for pure substances, we get from Eq. (3)

$$
=-\nabla \cdot L_{00} \nabla \frac{1}{T}
$$

Rearranging the formulae, results in the evolution equation for the temperature field

$$
\frac{\partial T}{\partial t}=\frac{-\nabla \cdot L_{00} \nabla \frac{1}{T}-\left(\frac{\partial f}{\partial \phi}-T \frac{\partial^{2} f}{\partial T \partial \phi}\right) \frac{\partial \phi}{\partial t}}{-T \frac{\partial^{2} f}{\partial T^{2}}}
$$

In our studies, we consider pure $\mathrm{Ni}$ in two phase states solid and liquid phase. The data and properties from [30] computed with the MD model Embeded Atom Method potential developed by Foiles et al. [31]. For the comparison to MD in section 4.1, the PFM simulations are calculated for isothermal undercooling conditions, so the free energy provides a constant term to the equation of motion (4): $\partial_{\phi} f=$ const $\cdot h^{\prime}(\phi)$. In this case, the temperature field is constant and Eq. (9) is not calculated. The temperature equation is calculated for the dendritic growth in section 4.2. 


\section{Data conversion from different atomistic methods to mesoscopic PFM}

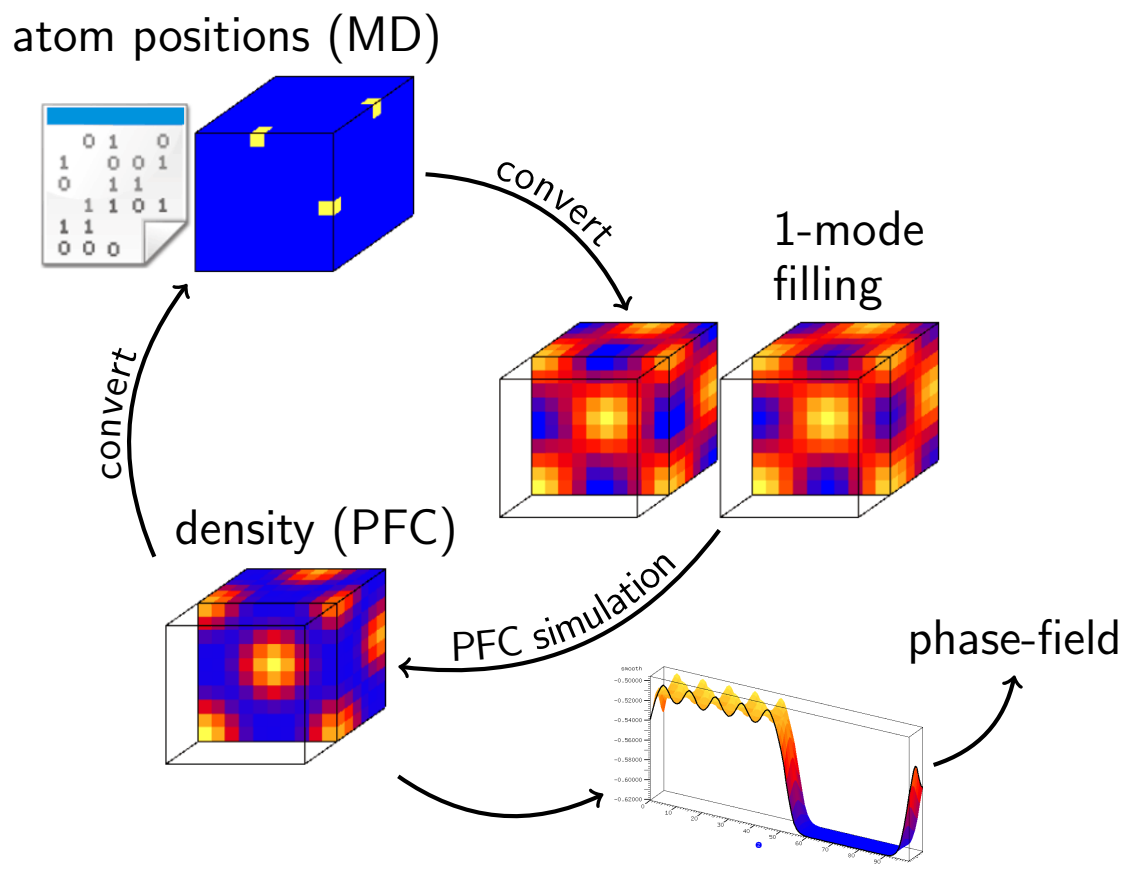

Figure 1: Conversion of atomic data from MD to PFC and from PFC to continuum data for PFM.

In order to compare the results of different atomistic models, we developed methods to convert data from different formats MD to PFC, vice versa, MD to PFM and $\mathrm{PFC}$ to PFM as illustrated in Fig 1. Discrete atom positions, as generated by MD are converted to atom-densities used for PFC by setting a normal distribution at the atom positions. This is very similar to the 1-mode filling method, where different crystallographic modifications, such as fcc and bcc, serve as initial density profiles.

Conversely, the atom positions can be extracted from local maxima of density profiles obtained by PFC. These atom positions can be used to analyze local structure using order parameters such as the local bond order parameter $q_{6} q_{6}$ [32].

In order to convert PFC to PFM, we exploit the free energy calculated out of the density profile, to extract a phase-field order parameter profile. The $q_{6} q_{6}$ order parameter from MD data can be applied to generate an appropriate diffuse interface profile.

With these conversion methods, we are able to compare atomistic properties of different methods, and transfer data from atomistic scales to simulate large microstructure formations on mesoscopic scales. 
melting temperature solid density

liquid density

latent heat

solid specific heat capacity

liquid specific heat capacity

thermal diffusivity

kinetic growth coefficient

interface free energy

interface width parameter

interface entropy density

$$
\begin{aligned}
& T_{m}=1748 \mathrm{~K} \\
& \rho^{s}(T)=\left(8901.6-0.20379 T-6.142 \cdot 10^{-5} T^{2}\right)^{\mathrm{kg} / \mathrm{m}^{3}} \\
& \rho^{\ell}(T)=\left(8992.3-0.66704 T-3.3161 \cdot 10^{-5} T^{2}\right)^{\mathrm{kg}} / \mathrm{m}^{3} \\
& L(T)=\left(-15980+324.75 T-8.1098 \cdot 10^{-2} T^{2}\right)^{\mathrm{J} / \mathrm{kg}} \\
& c_{v}^{s}(T)=\rho^{s}(T)\left(419.452+2.0388 \cdot 10^{-2} T\right)^{\mathrm{J}} / \mathrm{kgK}^{2} \\
& c_{v}^{\ell}(T)=\rho^{\ell}(T)\left(563.023-6.952 \cdot 10^{-2} T\right)^{\mathrm{J}} / \mathrm{kgK}^{2} \\
& \alpha=2.1 \cdot 10^{-7} \mathrm{~m}^{2} / \mathrm{s} \\
& k_{0}=0.319205 \mathrm{~m} / \mathrm{sK}, \epsilon_{k}=-0.196511, \delta_{k}=0.230331 \\
& \gamma_{0}=0.302 \mathrm{~J} / \mathrm{m}^{2}, \epsilon_{1}=0.10191, \epsilon_{2}=-0.00134, \epsilon_{3}=0.00876 \\
& \epsilon=4 \AA \\
& \gamma_{s \ell}=0.1728 \cdot 10^{-3} \mathrm{~J} / \mathrm{m}^{2} \mathrm{~K}
\end{aligned}
$$

Table 1: Parameters and functional expressions from MD composed for PFM simulations.

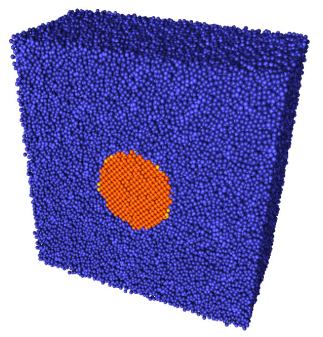

(a)

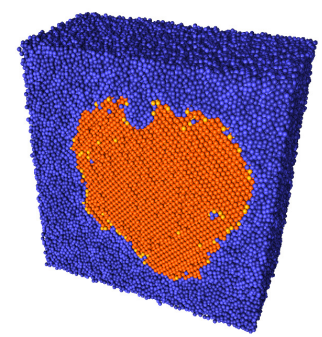

(b)

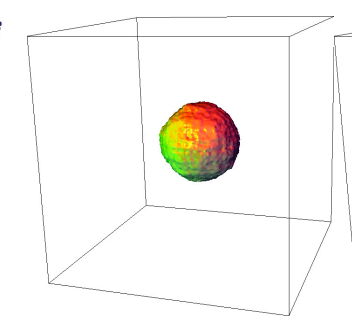

(c)

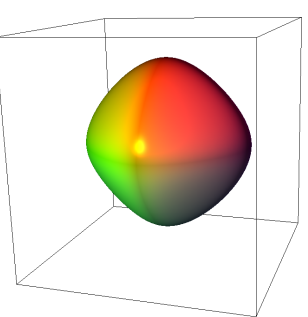

(d)

Figure 2: Snapshots of MD (a) and (b) and PFM (c) and (d) simulations at a temperature of $1550 \mathrm{~K}$. The evolution of the crystalline cluster of PFM in (c) and (d) is displayed for the same volume fractions as MD

\section{Simulation applications to metals}

\subsection{Comparison between MD and PFM structures on atomistic scale}

We start with initial $\mathrm{Ni}$ nuclei of $5 \mathrm{~nm}$ in diameter and simulate some hundreds picoseconds up to a size of $15 \mathrm{~nm}$ with the help of MD. The properties of Ni required in the PFM simulations were obtained from MD simulations [30, 33] using the EAM F85 model [31] and listed in Tab. 1. An anisotropic kinetic coefficient is modelled by

$$
\frac{k(\hat{n})}{k_{0}}=1-3 \epsilon_{k}+4 \epsilon_{k} Q+\delta_{k}(P+30 S)
$$

with $Q=n_{1}^{4}+n_{2}^{4}+n_{3}^{4}, P=n_{1}^{6}+n_{2}^{6}+n_{3}^{6}$ and $S=n_{1}^{2} n_{2}^{2} n_{3}^{2} . \hat{n}=\left(n_{1}, n_{2}, n_{3}\right)$ is the unit vector normal to the local interface. The orientational dependence of the interface free energy is described by a cubic harmonic expansion of the form

$$
\frac{\gamma(\hat{n})}{\gamma_{0}}=1+\epsilon_{1}\left(Q-\frac{3}{5}\right)+\epsilon_{2}\left(3 Q+66 S-\frac{17}{7}\right)+\epsilon_{3}\left(5 Q^{2}-16 S-\frac{94}{13} Q+\frac{33}{13}\right) .
$$

The initial nucleus and final MD atom cluster at $T=1550 \mathrm{~K}$ is shown in Fig. 2 in comparison with PFM growth structures. The nature of PFM smoothens the interface roughness, so that a direct comparison between MD and PFM is inapplicable. 


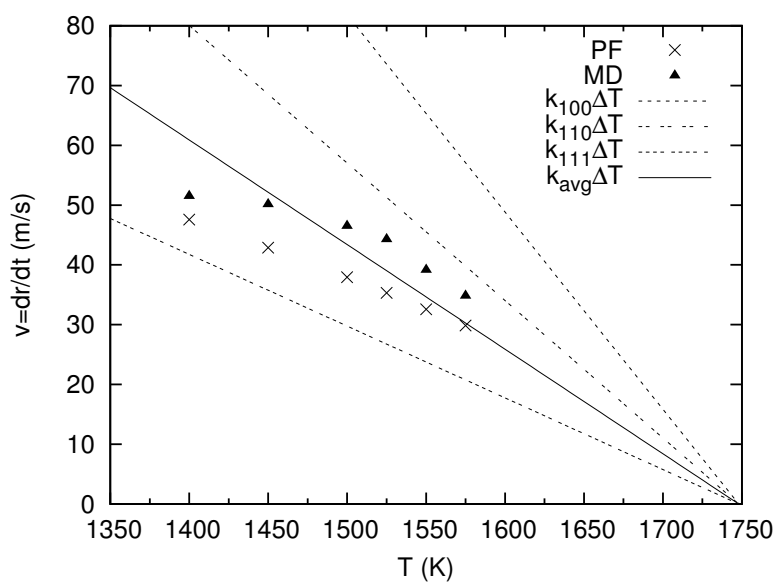

Figure 3: Asymptotic radial velocity versus temperature. Values of MD and PFM are obtained from the asymptotic limit.

Therefore, we assume an exponential velocity $\frac{\mathrm{d} r}{\mathrm{~d} t}$ of the interface over the approximated sphere radius where $r$ is calculated from the volume of the nuclei, under the assumption, that it is a sphere. Due to the fact that the MD computation is performed for an isothermal temperature, we also assume isothermal conditions for the PFM simulations.

Fig. 3 shows the PFM and MD simulation results for spherical nuclei and for planar growth with different crystal orientations. For a spherical nucleus, the theoretical kinetic $k_{\mathrm{avg}}=\int_{S^{2}} k(\hat{n}) \mathrm{d} A / \int_{S^{2}} 1 \mathrm{~d} A$ is expected, details in [34]. Due to the nature of PFM, a spherical nucleus forms 111-facets, so that $k_{\text {avg }}$ is an upper and $k_{111}$ a lower bound for the velocity.

\subsection{Iterative up-scaling method}

The continuum PFM has the property of simply scaling the length. However, the diffuse interface has a width of about 10 grid points, such that scaling by a factor of 2 does not disturb the interface significantly. We start with a nucleus, set in one corner of a simulation cube of $145^{3}$ grid points, with a numerical cell spacing of $\Delta x=1 \AA$ and assume trivial symmetry of a cubic crystal shape (see upper left of Fig. 4a). The initial temperature is set at $1450 \mathrm{~K}$. Before boundary effects occur, i. e. the nucleus or the temperature reache the boundary, the growth is interrupted. $\Delta x$ is doubled such that the physical size increases, but the simulation box remains at the same extensions, the nucleus is scaled to this box. The free area inside the domain is set by pre-defined values. The stability criteria of the numerical solution scheme allow an increase of the time step by a factor of 4 for each up-scaling step. This procedure can be continued for some time, however, it is bounded by the stability of the phase-field interface. We find, that for the parameter set of Tab. 1, the maximum grid point distance is $\Delta x=5 \AA$. For larger $\Delta x$, the interface becomes asymmetric [35].

\subsection{Hill tetrahedron construction}

A further upscaling can be achieved by enlarging the simulation box, so as to simulate larger crystals. We expect a symmetrical dendrite with 6 arms, where 3 of them lie in 


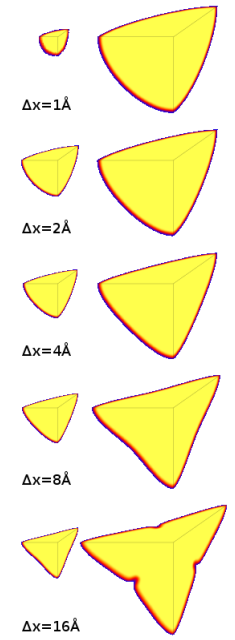

(a)

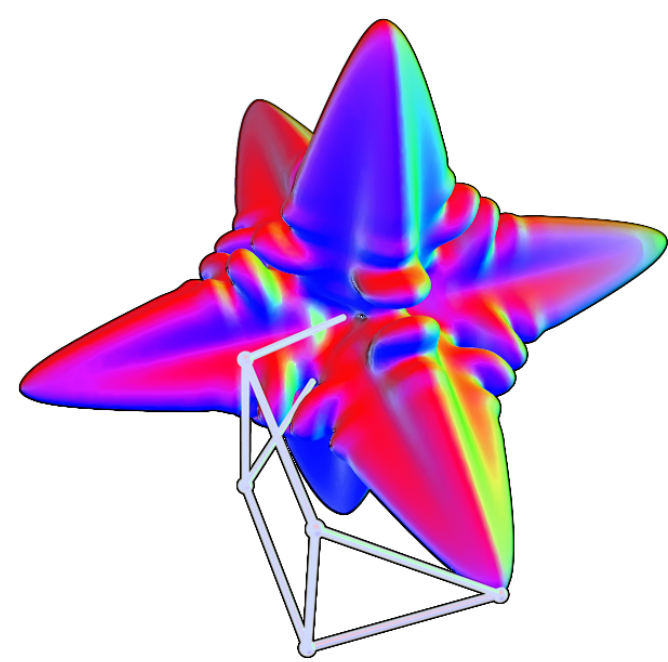

(b)

Figure 4: (a) Four iterative up-scaling steps of a dendritic crystal. Each row shows the different cell spacing. (b) Resulting dendrite and applied simulation box according to half of a Hill tetrahedron.

the simulation box. Based on this symmetry assumptions, the number of calculated grid points can be reduced. It is simple to see that one Hill tetrahedron contains the whole information of the symmetric crystal. Fig. 4b shows a dendrite composed of the part calculated in half of a Hill tetrahedron [36]. We remark that calculating only the symmetric part will always develop symmetric arms, even with an induced noise in the simulation.

\section{Phase-field crystal model for pure substances}

The phase-field crystal model (PFC) is represented by an atomic density field $\psi$. The dynamics is simulated dissipatively, according to the minimization of the free energy functional

$$
\mathcal{F}=\int \mathrm{d} \boldsymbol{r}\left\{\frac{\psi(\boldsymbol{r})}{2}\left(-\epsilon+\left(1+\nabla^{2}\right)^{2} \psi(\boldsymbol{r})\right)+\frac{1}{4} \psi(\boldsymbol{r})^{4}\right\}
$$

which leads to the evolution equation

$$
\frac{\partial \psi}{\partial t}=\nabla^{2}\left[\left(-\epsilon+\left(1+\nabla^{2}\right)^{2}\right) \psi+\psi^{3}\right]
$$

where $\epsilon$ corresponds to the undercooling.

As proposed in [37], we use an operator-splitting, semi-implicit Fourier-spectral method to solve the PFC equations which allows to easily incorporate different model formulations such as the $4^{\text {th }}$ and $8^{\text {th }}$-order models discussed in ([11], [13] and [38]). For the following comparison between hard spheres and PFC, we employ the model system given in Eq. (12).

As presented in [39] the wave number $q_{0}$ for the lowest free energy and the equilibrium densities $\bar{\psi}_{\ell}, \bar{\psi}_{s}$ for the liquid and solid phases, can be determined by means 
of a Monte Carlo-based n-mode expansion. Applying the wave number $q_{0}$, a crystal without boundary effects can be set in a simulation domain with periodic boundary conditions. In equilibrium state, we measure the interface energy [38] for different crystal directions by

$$
\gamma=\frac{1}{\Omega} \int_{\Omega} \mathrm{d} \mathbf{r}\left\{f-\left(f_{s} \frac{\psi-\bar{\psi}_{\ell}}{\bar{\psi}_{s}-\bar{\psi}_{\ell}}-f_{\ell} \frac{\psi-\bar{\psi}_{s}}{\bar{\psi}_{s}-\bar{\psi}_{\ell}}\right)\right\} .
$$

The initial setting is a one-mode approximation of the fcc-structure. The PFC simulations recover the expected interface energies $\gamma$ for the different crystal orientations. The $\{100\}$-direction has the highest energy and the $\{111\}$-direction has the lowest energy (Fig. 5 and [40]).

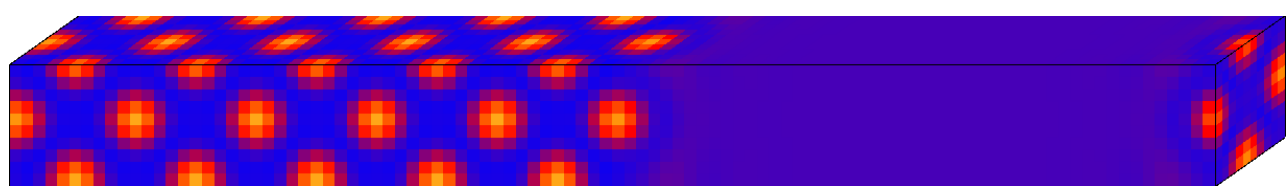

(a) density of an fcc crystal in $\{100\}$ direction

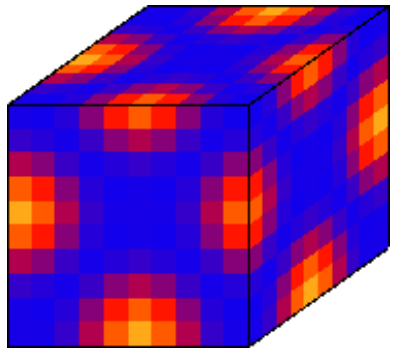

(b) $\{100\}: \gamma=1.134 e-04$

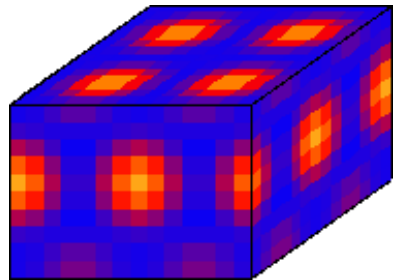

(c) $\{110\}: \gamma=7.637 e-05$

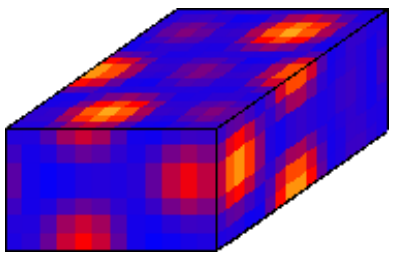

(d) $\{111\}: \gamma=5.677 e-05$

Figure 5: (a) Rod of 10 unit cells. (b)-(d) Dimensionless interface energy $\gamma$ for different crystal directions showing one unit cell.

\subsection{Colloids PFC simulations}

The PFC model can be interpreted as a time interpolation of atomic positions. Therefore it acts on a higher time scale than MD. If PFC is simulating atoms, it acts naturally on the atomistic length scale and can replace classical MD to deliver initial states for further scale-bridging simulations. However, it is not as much developed as MD. Jaatinen et al. have developed a quantitative PFC model for iron. Wu et al. have extended this approach fcc-structure metals like nickel in a non-quantitative manner. The main physics of PFC lies in its correlation function and potential. So an interpretation of the peaks as colloids, as done by Teeffelen et al. [41], is a valid step that increases the length scale. Fig. 6 shows an example of a grown fcc-structure. An extended model to Eq. (12) with a functional of the form

$$
\mathcal{F}=\int \mathrm{d} \boldsymbol{r}\left\{\frac{\psi(\boldsymbol{r})}{2}\left(B_{L}+B_{S}\left(2 \nabla^{2}+\nabla^{4}\right)\right) \psi(\boldsymbol{r})+\frac{v}{6} \psi(\boldsymbol{r})^{3}+\frac{1}{12} \psi(\boldsymbol{r})^{4}\right\}
$$

is numerically solved with $B_{L}=0.288725134, B_{S}=0.288675134$ and $v=0.658037006$ introduced in [42]. The simulation was started with a small cubic fcc-nucleus of 32 cells in a domain of 1024 cells in each direction with $\Delta x=0.72552$ and $\psi_{0}=-0.04$. 


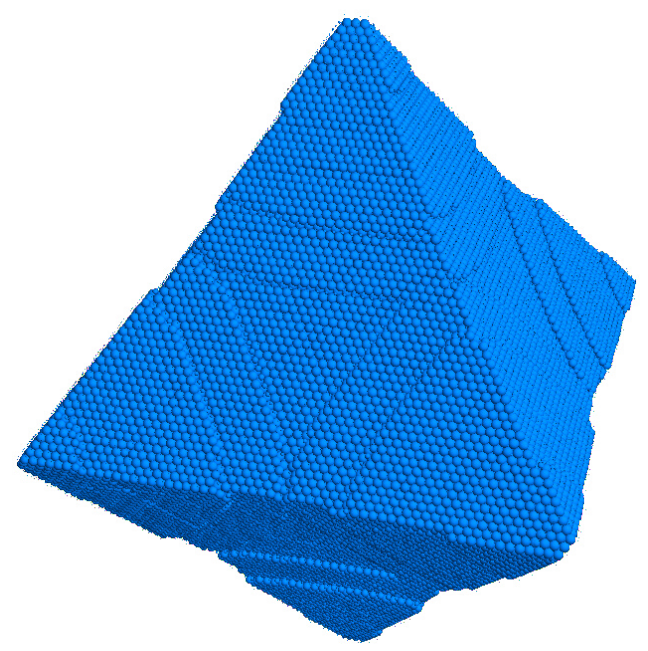

Figure 6: Atom position distribution of an fcc-structure computed with PFC

\section{Conclusion}

In this contribution we have introduced conversion and up-scaling methods to bridge the scales. The atomistic models, such as the MD and PFC model are of course, limited to the atomistic length scale, in order to computationally resolve atoms. Thus, greater structures can only be calculated in larger volume elements. This means, that the calculation effort dramatically increases to non feasible amounts. At this point the advantage of the continuum models, such as the PFM, becomes inevitable, as there is (almost) no physical limitation of the length scale. So the length scale can be set to the atomic level without difficulty.

To supply the necessary material quantities for microstructure simulations on the mesoscopic scale of several $\mu \mathrm{m}$, the atomistic models provide the parameters for the PFM. Alternatively the data are already input parameters in the atomistic model, or the model can be calculated from atomistic simulations, which was exemplarily shown by specifying the interface energy.

In section 4.1, we compare PFM with MD on an atomic level. Apart from the smoothing property of the PFM, the growth rates exhibit good agreements. The crystals do not grow faster than the average growth rate $k_{\text {avg }}$ and approach the growth rate $k_{111}$ after the development of $\{111\}$ facets.

In section 5 it is shown that the PFC model can also provide physical parameter for the PFM.

Due to the restrictions of the interface width with respect to numerical grid resolution, the very efficient up-scaling method presented in section 4.2 , reaches its limits such that an increase of the domain and hence computational effort is mandatory. Because of the symmetry properties of a dendrite, the domain, however, can be adapted in such a way, that only one Hill tetrahedron, and thus only $1 / 48$, has to be calculated. Both methods enable a calculation of a thermal dendrite on a mesoscopic scale, where ternary side arms start to develop.

The authors gratefully acknowledge the financial support by the German Research Foundation (DFG) in the Priority Program SPP 1296.

\section{REFERENCES}


1. Provatas N, Greenwood M, Athreya B, Goldenfeld N, and Dantzig J: International Journal of Modern Physics B 19(31) 4525-4566, 2005.

2. Provatas N, Dantzig J A, Athreya B, Chan P, Stefanovic P, Goldenfeld N, and Elder K R: JOM Journal of the Minerals, Metals and Materials Society 59(7) 83-90, 2007.

3. Hoyt J J, Sadigh B, Asta M, and Foiles S M: Acta materialia 47(11) 3181-3187, 1999.

4. Hoyt J J, Asta M, and Karma A: Materials Science and Engineering R 41 121163, 2003.

5. Tupper P F and Grant M: EPL (Europhysics Letters) 81 40007, 2008.

6. Kadau K, Germann T C, and Lomdahl P S: International Journal of Modern Physics C 17 1755-1761, 2006.

7. Frenkel D and Smit B: Understanding molecular simulation: from algorithms to applications. Academic Press, 2002.

8. Allen M P and Tildesley D J: Computer simulation of liquids. Oxford university press, 1989.

9. Leach A R: Molecular modelling: principles and applications. Pearson Education, 2001.

10. Mishin Y, Asta M, and Li J: Acta Materialia 58(4) 1117-1151, 2010.

11. Elder K R and Grant M: Physical Review E 70(5) 51605, 2004.

12. Elder K R, Provatas N, Berry J, Stefanovic P, and Grant M: Phys. Rev. B 75(6) 64107, 2007.

13. Jaatinen A, Achim C V, Elder K R, and Ala-Nissila T: Physical Review E 80 $031602,2009$.

14. Greenwood M, Rottler J, and Provatas N: Physical Review E 83(3) 031601, 2011.

15. Tóth G I, Tegze G, Pusztai T, and Gránásy L: Phys. Rev. Lett. 108 025502, 2012.

16. Wu K A, Adland A, and Karma A: Arxiv preprint arXiv:1001.1349 2010.

17. Chen L Q: Annual Review of Materials Research 32(1) 113-140, 2002.

18. Boettinger W J, Warren J A, Beckermann C, and Karma A: Annual Review of Materials Research 32(1) 163-194, 2002.

19. Steinbach I: Modelling and Simulation in Materials Science and Engineering $\mathbf{1 7}(7)$ 073001, 2009.

20. Kobayashi R: Physica D: Nonlinear Phenomena 63(3) 410-423, 1993.

21. Warren J A and Boettinger W J: Acta Metallurgica et Materialia 43(2) 689-703, 1995.

22. Nestler B, Garcke H, and Stinner B: Physical Review E 71(4) 041609, 2005.

23. Nestler B and Wheeler A A: Physica D: Nonlinear Phenomena 138(1-2) 114-133, 2000.

24. Folch R and Plapp M: Physical Review E 72(1) 011602, 2005.

25. Eiken J, Böttger B, and Steinbach I: Physical Review E 73(6) 066122, 2006.

26. Choudhury A and Nestler B: Physical Review E 85(2) 021602, 2012.

27. Moelans N, Blanpain B, and Wollants P: Calphad 32(2) 268-294, 2008.

28. Takaki T, Hisakuni Y, Hirouchi T, Yamanaka A, and Tomita Y: Computational Materials Science 45(4) 881-888, 2009.

29. Gránásy L, Pusztai T, and Warren J A: Journal of Physics: Condensed matter 16 R1205, 2004.

30. Rozas R E and Horbach J: to be submitted.

31. Foiles S M: Physical Review B 32(6) 3409, 1985.

32. Zykova-Timan T, Rozas R E, Horbach J, and Binder K: Journal of Physics: Condensed Matter 21 464102, 2009.

33. Rozas R E and Horbach J: Europhysics Letters 93 26006, 2011.

34. Berghoff M, Selzer M, and Nestler B: The Scientific World Journal in press.

35. Karma A and Rappel W J: Physical Review E 53(4) 3017-3020, 1996. 
36. Berghoff M, Selzer M, Choudhury A, and Nestler B: Journal of Computational Methods in Science and Engineering in press.

37. Cheng M and Warren J A: Journal of Computational Physics 227(12) 6241-6248, 2008.

38. Wu K A and Karma A: Physical Review B 76(18) 184107, 2007.

39. Oettel M: Journal of Physics: Condensed Matter 24(46) 464124, 2012.

40. Oettel M, Dorosz S, Berghoff M, Nestler B, and Schilling T: Physical Review E 86(2) 021404, 2012.

41. van Teeffelen S, Backofen R, Voigt A, and Löwen H: Physical Review E $79(5)$ 051404, 2009.

42. Tegze G, Gránásy L, Tóth G I, Podmaniczky F, Jaatinen A, Ala-Nissila T, and Pusztai T: Physical Review Letters 103(3) 035702, 2009. 